\title{
The Law of the Iterated Logarithm in Analaysis
}

\author{
Santosh Ghimire \\ Department of Engineering Science and Humanities, Institute of Engineering, Central Campus, Pulchowk, \\ Tribhuvan University, Nepal \\ Corresponding Email: santoshghimire@ioe.edu.np
}

\begin{abstract}
In this paper, we first discuss the history of the law of the iterated logarithm. We then focus our discussion on how it was introduced in analysis. Finally we mention different types of law of the iterated logarithm and state some of the recent developments.
\end{abstract}

In order to discuss the history and developments of law of the iterated logarithm, some definitions and theorems are in order:

\section{Definition 1 [Normal Numbers]}

Suppose that $\mathrm{N}$ takes values in $[0,1)$ and consider its decimal and dyadic expansion as:

$$
N=\sum_{n=1}^{\infty} \frac{X_{n}}{10^{n}} ; X_{n} \in\{0,1,2, \ldots, 9\}, N=\sum_{n=1}^{\infty} \frac{X_{n}}{2^{n}} ; X_{n} \in\{0,1\}
$$

Now for a fixed $\mathrm{k}, 0 \leq \mathrm{k} \leq 9$, let $\omega_{\mathrm{k}}^{(\mathrm{n})}(\mathrm{N})$ denote the number of digits among the first $\mathrm{n}$-digits of $\mathrm{N}$ that are equal to $\mathrm{k}$. Then $\frac{\omega_{\mathrm{k}}^{(\mathrm{n})}(\mathrm{N})}{\mathrm{n}}$ is the relative frequency of the digit $\mathrm{k}$ in the first $\mathrm{n}$ places and thus the limit $\lim _{\mathrm{n} \rightarrow \infty} \frac{\omega_{\mathrm{k}}^{(\mathrm{n})}(\mathrm{N})}{\mathrm{n}}$ is the frequency of the $\mathrm{k}$ in $\mathrm{N}$. Then the number $\mathrm{N}$ is called the normal to the base 10 if and only if this limit exists for each $\mathrm{k}$ and is equal to $\frac{1}{10}$. Similarly, the number $\mathrm{N}$ is called the normal to base 2 if and only if the limit exists and is equal to $\frac{1}{2}$.

\section{Definition 2 [Lacunary Series]}

A real trigonometric series with partial sums $S_{m}(\theta)=\sum_{k=1}^{m}\left(a_{k} \cos n_{k} \theta+b_{k} \cos n_{k} \theta\right)$ which has $\frac{\mathrm{n}_{\mathrm{k}+1}}{\mathrm{n}_{\mathrm{k}}} \geq q>1$ is called q-lacunary series.

In the above definition, the condition $\frac{\mathrm{n}_{\mathrm{k}+1}}{\mathrm{n}_{\mathrm{k}}} \geq q>1$ is called the gap condition (lacunarity condition) which states that the sequence $\left\{n_{k}\right\}$ increases at least as rapidly as a geometric progression whose common ratio is bigger than 1 . Lacunary series exhibit many of the properties of partial sums of independent random variables. In the modern probability theor, lacunary series are called 'weakly dependent' random variables.

Keywords and phrases: law of the iterated logarithm, lacunary series, normal numbers, Rademacher's function, Borel's theorem 


\section{Definition 3 [Rademacher function]}

The functions $\left\{r_{k}\right\}_{k=1}^{\infty}$ defined on $[0,1)$ by $r_{k}(x)=\operatorname{sgn}\left(\sin 2^{k} \pi x\right)$ where $\operatorname{sgn}$ is given by $\operatorname{sgn}(x)=1$ if $x \leq 0$ and $\operatorname{sgn}(x)=-1$ if $x<0$ are called the Rademacher functions.

We note that $r_{k}$ alternates +1 and -1 on the dyadic intervals of the generation $k$. Moreover, $r_{k}^{\prime} s$ are independent, identically distributed random variables with mean zero and variance one.

\section{Theorem 1 [Borel]}

If $N_{n}(t)$ denote the number of occurences of the digit 1 in the first $n$-places of the binary expansion of a number $t \in[0,1)$, then $\lim _{n \rightarrow \infty} \frac{N_{n}(t)}{n}=\frac{1}{2}$ for a.e. $t$ in Lebesgue measure.

The first law of the iterated logarithm (LIL) was introduced in the probability theory in attempting to perfect the Borel's theorem (stated earlier) on normal numbers. Precisely, the first LIL was introduced to obtain the exact rate of convergence in the Borel's theorem where number of mathematicians obtained the different rates of convergence and A. Khintchine was the one who obtained the desired rate. To describe this, let $X_{n}$ denote the random variable which takes values \pm 1 with probability $\frac{1}{2}$ and we take unit interval with Lebesgue measure as a probability space. Then we can write $\mathrm{X}_{\mathrm{n}}(\mathrm{t})=2 b_{n}(t)-1$, where $\mathrm{b}_{\mathrm{n}}$ is the $\mathrm{n}^{\text {th }}$ digit in the binary expansion of $t \in[0,1)$. Let $S_{n}=\sum_{i=1}^{n} X_{i}$. Under this context, following result were obtained:

- Hausdorff (1913) obtained $\left|S_{n}\right|=O\left(n^{\frac{1}{2}+\varepsilon}\right)$ a.e. for any $\varepsilon>0$.

- Hardy and Littlewood (1914) obtained $\left|S_{n}\right|=O(\sqrt{n \operatorname{logn}})$ a.e.

- Khintchine (1923) obtained $\left|S_{n}\right|=O(\sqrt{n \log \log n})$ a.e.

In 1924, [1] obtained the definite answer to the size of the deviation in the Borel's theorem and his result is given by:

\section{Theorem 2 [Khintchine, 1924]}

If $\mathrm{N}_{\mathrm{n}}(\mathrm{t})$ denote the number of occurences of the digit 1 in the first n-places of the binary expansion of a number $t \in[0,1)$, then for a.e $t$, we have,

$$
\limsup _{n \rightarrow \infty} \frac{\left|S_{n}\right|}{\sqrt{2 n \log \log n}}=1 \text {. }
$$

This result is popularly known as Khintchine's law of the itetrated logarithm and is the first LIL result in the probability theory. So Khintchine's LIL provides the size of the deviation in terms of expected mean and the deviation is of order $\sqrt{1 / 2 \mathrm{n} \log \log n}$. Because of the factor loglogn (iteration of $\log$ ) in the deviation, Khintchine's law is popularly known as law of the iterated logarithm. We note that Borel's theorem immediately follows from Khintchine's result. A few years later, the result of Khintchine was generalized by [5] to a wide class of independent random variables. Kolmogorov's LIL which in the words of K. L. Chung "is a crowning achievement in the classical probability theory". We now state the Kolmogorov's celebrated law of the iterated logarithm: 
Theorem 3 [Kolmogorov, 1929]

Let $S_{n}=\sum_{k=1}^{n} X k$ where $X_{k}$ is a sequence of real valued independent random variables. Let $s_{n}$ be the variance of $S_{n}$. Suppose that $S_{n} \rightarrow \infty$ and $\left|X_{n}\right|^{2} \geq \frac{K_{n} s_{n}}{\log \log \left(e^{e}+s_{n}^{2}\right)}$ some sequence of constants $K_{n} \rightarrow 0$. Then, almost surely,

$$
\limsup _{n \rightarrow \infty} \frac{S_{n}(\omega)}{\sqrt{2 s_{n} \log \log s_{n}^{2}}}=1 .
$$

We remark that in the above theorem, the mean of $\mathrm{S}_{\mathrm{n}}$ is zero and $\sqrt{\mathrm{n}}$ is the standard deviation of $\mathrm{S}_{\mathrm{n}}$. So Kolmogorov's LIL provides the size of partial sum

of independent random variable from its expected mean and the size is approximated in terms of standard deviation.

Let us apply Kolmogorov's LIL in a random walk to estimate the size of the walk in the long run. Consider the Rademacher functions, $\left\{\mathrm{r}_{\mathrm{k}}\right\}_{\mathrm{k}=1}^{\infty}$. Set,

$$
f_{1}(x)=r_{1}(x), f_{2}(x)=r_{1}(x)+r_{2}(x), \ldots, f_{n}(x)=r_{1}(x)+r_{2}(x)+\cdots+r_{n}(x)
$$

and so on.

Clearly, $\left\{\mathrm{f}_{\mathrm{n}}(\mathrm{x})\right\}$ defines a random walk. In this random walk, we move 1 unit to the right if $\mathrm{r}_{\mathrm{i}}(\mathrm{x})=1$ and to the left if $\mathrm{r}_{\mathrm{i}}(\mathrm{x})=-1$. Moreover, $\left\{\mathrm{f}_{\mathrm{n}}\right\}$ satisfies all the assumptions of Kolmogorov's theorem. So by Kolmogorov's LIL, we have,

$$
\limsup _{n \rightarrow \infty} \frac{\left|f_{n}(x)\right|}{\sqrt{2 n \log \log n}} \leq 1 \text {. }
$$

For $\varepsilon>0$, this gives us $\left|f_{n}(x)\right| \leq(1+\varepsilon) \sqrt{2 n \log \log n}$ for $n$ large. Here, the worst bound for the function $f_{n}(x)$ is $n$ i.e. $\left|f_{n}(x)\right| \leq n$. Thus, Kolmogorov's LIL gives the sharper asymptotic estimate, $\left|f_{n}(x)\right| \leq(1+\varepsilon) \sqrt{2 n \log \log n}$. For sufficiently large $n$, the factor $\sqrt{2 n \log \log n}$ is much smaller than $\mathrm{n}$. This shows that in the long run the walker will fluctuate in between $-\sqrt{2 n \log \log n}$ and $\sqrt{2 \mathrm{n} \log \log \mathrm{n}}$.

Over the years, people have obtained the analog the Kolmogorov's result in the various settings of analysis. Some of the existing settings are lacunary trigonometric series, martingales, harmonic functions to name just a few. But the first LIL in analysis was obtained in the setting of lacunary trigonometric series. The law of the itetrated logarithm in the lacunary setting was first obtained by [8].

\section{Theorem 4 [R. Salem and A. Zygmund, 1950]}

Suppose that $S_{n}$ is a q-lacunary series and the $n_{k}$ are positive integers. Set $B_{n}^{2}=\sum_{k=1}^{n}\left(\left|a_{k}\right|^{2}+\right.$ $\left.\left|b_{k}\right|^{2}\right)$ and $M_{n}=\max _{1 \leq k \leq n}\left(\left|a_{k}\right|^{2}+\left|b_{k}\right|^{2}\right)^{1 / 2}$. Suppose also that $B_{n} \rightarrow \infty$ and $S_{n}$ satisfies the Kolomogorov-type condition: $M_{n}^{2} \leq K_{n} \frac{B_{n}^{2}}{\log \log \left(e^{e}+B_{n}^{2}\right)}$ for some sequence of numbers $K_{n} \downarrow 0$. Then

$$
\limsup _{n \rightarrow \infty} \frac{S_{n}(\theta)}{\sqrt{2 B_{n}^{2} \log \log B_{n}}} \leq 1
$$

for almost every $\theta \epsilon T$, unit circle. 
This result of Salem and Zygmund is the first law of the iterated logarithm in analysis. Note that $\int_{-\pi}^{\pi} S_{m}(x)=0$. This means that the mean of partial sum is zero. Again,

$$
\begin{aligned}
\sigma^{2}=\operatorname{Variance}\left(\mathrm{S}_{\mathrm{m}}(\mathrm{x})\right) & =\frac{1}{2 \pi}\left[\int_{-\pi}^{\pi} \mathrm{S}_{\mathrm{m}}^{2}(\mathrm{x}) \mathrm{dx}-\left(\int_{-\pi}^{\pi} \mathrm{S}_{\mathrm{m}}(\mathrm{x})\right)^{2}\right] \\
& =\frac{1}{2 \pi} \int_{-\pi}^{\pi} \mathrm{S}_{\mathrm{m}}^{2}(\mathrm{x}) \mathrm{dx}-0 \\
& =\frac{1}{2 \pi} \int_{-\pi}^{\pi}\left[\sum_{\mathrm{k}=1}^{\mathrm{m}}\left(\mathrm{a}_{\mathrm{k}} \cos _{\mathrm{k}} \mathrm{x}+\mathrm{b}_{\mathrm{k}} \sin \mathrm{n}_{\mathrm{k}} \mathrm{x}\right)\right]^{2} \mathrm{dx} \\
& =\frac{1}{2 \pi} \int_{-\pi}^{\pi} \sum_{\mathrm{k}=1}^{\mathrm{m}}\left[a_{k}{ }^{2} \cos ^{2}\left(\mathrm{n}_{\mathrm{k}} \mathrm{x}\right)+b_{k}{ }^{2} \sin ^{2}\left(\mathrm{n}_{\mathrm{k}} \mathrm{x}\right)\right] \mathrm{dx} \\
& =\frac{1}{2} \sum_{\mathrm{k}=1}^{\mathrm{m}}\left(a_{k}{ }^{2}+{b_{k}}^{2}\right)
\end{aligned}
$$

Hence,

$$
\sigma=\mathrm{B}_{\mathrm{m}}=\sqrt{\frac{1}{2} \sum_{\mathrm{k}=1}^{\mathrm{m}}\left(a_{k}^{2}+b_{k}^{2}\right)}
$$

This shows that $\mathrm{B}_{\mathrm{m}}^{2}$ is the variance of patial sums. So the theorem gives us the upper bound for the size of oscillation of partial sums from its expected mean and the order of the size depends on the size of standard deviation. Salem and Zygmund assumed $\mathrm{n}_{\mathrm{k}}$ to be positive integers and they only obtained the upper bound. Erdös and Gál (1955) were the first one to make progress towards the other inequality. They obtained the following result for a particular form of lacunary series given by the following theorem.

Theorem 5 [Erdös and Gál, 1955] Suppose $S_{m}(\theta)=\sum_{k=1}^{m} \exp \left(i n_{k} \theta\right)$ is a q-lacunary series and $\mathrm{n}_{\mathrm{k}}$ is are integers. Then,

$$
\limsup _{m \rightarrow \infty} \frac{s_{m}(\theta)}{\sqrt{2 m \log \log m}}=1
$$

for almost every $\theta \in \mathrm{T}$, unit circle.

A few years later, [3] gave the complete analouge of Kolmogorov's LIL in this setting. This result was the part of her Ph.D. thesis.

Theorem 6 [M. Weiss, 1959] Suppose $S_{m}(\theta)=\sum_{k=1}^{m}\left(a_{k} \cos n_{k} \theta+b_{k} \sin n_{k} \theta\right)$ is a q-lacunary series. Set $B_{m}^{2}=\sum_{k=1}^{m}\left(\left|a_{k}\right|^{2}+\left|b_{k}\right|^{2}\right)$ and $M_{m}=\max _{1 \leq k \leq m}\left(\left|a_{k}\right|^{2}+\left|b_{k}\right|^{2}\right)^{1 / 2}$. Suppose also that $B_{m} \rightarrow \infty$ and $S_{m}$ satisfies the Kolomogorov-type condition: $M_{m}^{2} \leq K_{m} \frac{B_{m}^{2}}{\log \log \left(e^{e}+B_{m}^{2}\right)}$ for some sequence of numbers $K_{m} \downarrow 0$. Then

$$
\limsup _{\mathrm{m} \rightarrow \infty} \frac{\mathrm{S}_{\mathrm{m}}(\theta)}{\sqrt{2 \mathrm{~B}_{\mathrm{m}}^{2} \log \log \mathrm{B}_{\mathrm{m}}}}=1
$$

for almost every $\theta \in \mathrm{T}$, unit circle.

Next, we discuss another law of the iterated logarithm introduced by [8]. In this LIL they considered tail sums of lacunary series instead of $n^{\text {th }}$ partial sums. 
Theorem 7 [R. Salem and A. Zygmund, 1950]

Suppose a q-lacunary series $\widetilde{\mathrm{S}_{\mathrm{N}}}(\theta)=\sum_{\mathrm{k}=\mathrm{N}}^{\infty}\left(\mathrm{a}_{\mathrm{k}} \cos \mathrm{n}_{\mathrm{k}} \theta+\mathrm{b}_{\mathrm{k}} \sin \mathrm{n}_{\mathrm{k}} \theta\right)$ where $\mathrm{c}_{\mathrm{k}}{ }^{2}=\mathrm{a}_{\mathrm{k}}{ }^{2}+\mathrm{b}_{\mathrm{k}}{ }^{2}$ satisfies $\sum_{\mathrm{k}=1}^{\infty} \mathrm{c}_{\mathrm{k}}{ }^{2}<\infty$. Define $\widetilde{\mathrm{B}_{\mathrm{N}}}=\left(\frac{1}{2} \sum_{\mathrm{k}=1}^{\infty} \mathrm{c}_{\mathrm{k}}{ }^{2} \cdot\right)^{\frac{1}{2}}$ and $\widetilde{\mathrm{M}_{\mathrm{N}}}=\max _{\mathrm{k} \geq \mathrm{N}}\left|\mathrm{c}_{\mathrm{k}}\right|$. Suppose that $\widetilde{\mathrm{B}_{1}}<\infty$ and $\widetilde{\mathrm{M}}_{\mathrm{N}}^{2} \leq \mathrm{K}_{\mathrm{N}}\left(\frac{\widetilde{\mathrm{B}_{N}^{2}}}{\log \log \frac{1}{\widetilde{\mathrm{B}_{\mathrm{N}}}}}\right)$ for some sequence of numbers $\mathrm{K}_{\mathrm{N}} \downarrow 0$ as $\mathrm{N} \rightarrow \infty$. Then,

for almost every $\theta \epsilon \mathrm{T}$, unit circle.

$$
\limsup _{\mathrm{m} \rightarrow \infty} \frac{\widetilde{\mathrm{S}_{\mathrm{N}}}(\theta)}{\sqrt{2 \widetilde{\mathrm{B}}_{\mathrm{N}}^{2} \log \log \frac{1}{\widetilde{\mathrm{B}}_{\mathrm{N}}}}} \leq 1
$$

In the above theorem, Salem and Zygmund only obtained the upper bound. In fact they only stated the above result in their paper. In 2012, [9] obtained the lower bound in their result. Their result is:

\section{Theorm 8 [S. Ghimire and C.N. Moore, 2012]}

Let $S_{m}(x)=\sum_{k=1}^{m} a_{k} \cos \left(2 \pi n_{k} x\right)$ be a partial sum of a q-lacunary series where $\frac{n_{k+1}}{n_{k}} \geq q>1$ and $\sum_{\mathrm{k}=1}^{\infty} \mathrm{a}_{\mathrm{k}}^{2}<\infty$. Assume that $\max _{\mathrm{k} \geq \mathrm{N}} \mathrm{a}_{\mathrm{k}}^{2}=\mathrm{o}\left(\frac{\frac{1}{2} \sum_{\mathrm{k}=\mathrm{N}}^{\infty} \mathrm{a}_{\mathrm{k}}^{2}}{\log \log \frac{1}{\sqrt{\frac{1}{2} \sum_{\mathrm{k}=\mathrm{N}}^{\infty} \mathrm{a}_{\mathrm{k}}^{2}}}}\right)$. Then for a.e. $\mathrm{x}$,

$$
\limsup _{\mathrm{n} \rightarrow \infty} \frac{\left|\sum_{\mathrm{k}=\mathrm{n}}^{\infty} \mathrm{a}_{\mathrm{k}} \cos \left(2 \pi \mathrm{n}_{\mathrm{k}} \mathrm{x}\right)\right|}{\sqrt{2 \frac{1}{2} \sum_{\mathrm{k}=\mathrm{n}}^{\infty} \mathrm{a}_{\mathrm{k}}^{2} \log \log \frac{1}{\frac{1}{2} \sum_{\mathrm{k}=\mathrm{n}}^{\infty} \mathrm{a}_{\mathrm{k}}^{2}}}} \geq 1 \text {. }
$$

Another type of LIL in the case of independent random variable was introduced by [2]. His LIL is,

\section{Theorem 9 [Chung, 1948]}

Let $\left\{X_{n}, n \geq 1\right\}$ be a sequence of indpendent identically distributed random variables with common distribution $\mathrm{F}$ with zero mean and variance $\sigma^{2}$, and with finite third moment $\mathrm{E}\left(|\mathrm{X}|^{3}\right)<$ $\infty$. Then $\liminf \sqrt{\frac{\operatorname{loglogn}}{n}} \max _{1 \leq j \leq n}\left|S_{j}\right|=\frac{\sigma \pi}{\sqrt{8}}$ with probability 1 .

A law of the iterated logarithm in the case of periodic function has been introduced by [10].

\section{Theroem 10 [C.N. Moore and X. Zhang, 2012]}

Suppose $\mathrm{f}$ is a Dini continuous function on $\mathbf{R}^{\mathrm{n}}$ with the property that $\mathrm{f}(\mathrm{x})=0$ whenever any coordinate of $\mathrm{x}$ is an integer, and $\int_{\mathrm{Q}} \mathrm{f}(\mathrm{x}) \mathrm{dx}=0$ whenever $\mathrm{Q} \in \mathrm{F}_{0}$. Let $\left\{\mathrm{n}_{\mathrm{k}}\right\}$ be a sequence of positive numbers satisfying the lacunarity condition $\frac{n_{k+1}}{n_{k}} \geq q>1$ and $\left\{c_{k}\right\}$ be a sequence in $R^{n}$. Then there exists a constant $\mathrm{C}$, depending only on $\mathrm{n}, \mathrm{q}$, and the quantity $\int_{0}^{1} \frac{\omega(\delta)}{\delta} \mathrm{d} \delta$ such that for any sequence of numbers $\left\{a_{k}\right\}$ with $A_{m}=\sqrt{\sum_{k=1}^{m}\left|a_{k}\right|^{2}} \rightarrow \infty$ as $m \rightarrow \infty$, we have, 


$$
\lim _{\mathrm{m} \rightarrow \infty} \sup \frac{\left|\sum_{\mathrm{k}=1}^{\mathrm{m}} \mathrm{a}_{\mathrm{k}} \mathrm{f}\left(\mathrm{n}_{\mathrm{k}} \mathrm{x}+\mathrm{c}_{\mathrm{k}}\right)\right|}{\sqrt{\mathrm{A}_{\mathrm{m}}^{2} \log \log \mathrm{A}_{\mathrm{m}}^{2}}} \leq \mathrm{C}
$$

a.e.

There are many contexts in analysis where we have law of the iterated logarithm results such as dyadic martingales, Rademacher functions, Bloch functions, harmonic functions to name just a few. Readers are suggested to read the survey article on law of the iterated logarithm by [4].

\section{References}

[1] Khintchine, A. (1924). Über einen Satz der Wahrscheinlichkeitsrechnung, Fundamentall Mathematica, 6, 9-20.

[2] Chung, K. L. (1974). A Course in probability theory, Academic Press, New York.

[3] Weiss, M. (1959). The law of the iterated logarithm for lacunary trigonometric series, Transaction of American Mathematical Society, 91, 444-469.

[4] Bingham, N. H. (1986). Variants on the law of the iterated logarithm, Bulletin of the London Mathematical Society, Volume 18, Issue 5, 433-467.

[5] Kolmogorov, N. (1929). Uber des Gesetz des iterierten Logarithmus, Mathematische Annalen, 101, 126139

[6] Erdös, P. and Gál, I. S. (1955). On the law of the iterated logarithm, Nederl. Akad. Wetensch. Proc. Ser. A, 58, 65-84.

[7] Ba nelos, R. and Moore, C. N. (1991). Probabilistic Behavior of Harmonic Functions, Birkhauser Verlag.

[8] Salem, R. and Zygmund, A. (1950). La loi du logarithme itere pour les series trigonometriques lacunaires, Bulletin of Science and Mathematics, 74, 209-224.

[9] Ghimire, S. and Moore, C. N. (2012). The law of the iterated logarithm for tail sums, Proceeding of American Mathematical Society (to appear).

[10] Moore, C. N. and Zhang, X. (2012). A law of the iterated logarithm for general lacunary series Colloq. Math., 126, 95-103. 